\title{
THE MORPHOLOGICAL EVOLUTION OF FIELD GALAXIES
}

\author{
RICHARD S. ELLIS \\ Institute of Astronomy \\ Madingley Road, Cambridge CB3 OHA, UK \\ (rse@mail.ast.cam.ac.uk)
}

\begin{abstract}
I review two observational programs which, together, promise to unravel the detailed astrophysical evolution of normal field galaxies over the last 5-7 Gyr. Systematic ground-based spectroscopy of faint galaxies have revealed an increasing faint end slope for the luminosity function with redshift. The trend is strongest for galaxies undergoing intense star-formation. Deep images taken with the repaired HST can be used to count galaxies as a function of morphological type. Regular "Hubble sequence" galaxies follow the no-evolution prediction, but irregular/peculiar sources have a steeper count slope and provide the excess population. Although the overlap between the spectral and HST samples is currently small, plans to merge similar datasets should reveal the physical explanation for the demise of star formation in faint blue galaxies since $z \simeq 0.5-1$.
\end{abstract}

\section{Introduction}

Multi-object spectroscopy on 4-m telescopes has certainly transformed our understanding of the faint galaxy population. Controlled surveys to limiting magnitudes of $b_{J}=21.5$ (Broadhurst et al., 1988), $b_{J}=22.5$ (Colless et al., 1990,1993 ) and $B=24$ (Cowie et al., 1992; Glazebrook et al., 1994) have revealed a consistent trend in the redshift distribution, $N(z)$, which can only be logically explained via an increase in the absolute normalisation of the field galaxy luminosity function (LF) with redshift. The absence of an excess tail at low $z$ provides a very strong constraint on any uncertainties in the local LF (c.f. McGaugh, 1994). As the blue passband is particularly sensitive to small changes in star formation, the absence of a dominant high $z$ tail also restricts strong evolution in massive galaxies. In 1988, Broadhurst 
et al. proposed that the most likely explanation is a recent decline in the number and/or star formation rate of sub- $\mathrm{L}^{*}$ galaxies. They presented an empirical model which predicts a flattening with time in the faint end slope of the optical LF.

Babul and Rees (1992) suggested the faint blue excess is associated with a new dwarf population, recently "active" in star formation, but subsequently fading out of view. Broadhurst et al. (1990) proposed that distant star forming "sub-units" may slowly merge to form more massive $L^{*}$ galaxies. Both hypotheses can explain the empirical trends observed, but with different implications. Only in the latter case is the blue excess clearly related to the evolutionary history of disk galaxies like the Milky Way.

Might the blue galaxies be morphologically distinguishable from their "quiescent" counterparts? From the ground, a typical 22nd magnitude galaxy is a 1.5 arcsecond blur. Despite valiant attempts in sub-arcsecond seeing (Giraud, 1992; Colless et al., 1994), the morphologies of typical faint systems remained unclear. Colless et al.) present tentative evidence for the occurrence of multiple, presumed merging, systems with enhanced star formation revealed from spectral measures. However, those authors admit the ambiguity of distinguishing between genuine mergers and irregular systems with patchy $\mathrm{H}$ II regions.

Here I review recent progress on redshift surveys and high resolution imaging of the faint galaxy population. An extensive new redshift survey, the Autofib survey (Heyl, 1994; Ellis et al., 1994) verifies, for the first time, that the faint end slope of the LF is steepening with increasing redshift as predicted by Broadhurst et al. (1988). The steepening is attributable to an increased abundance of star-forming late type galaxies. The repair of Hubble Space Telescope (HST) allows galaxy counts to be determined as a function of morphological type. Again, a population of irregular systems appears to evolve more rapidly than the remainder. Although the overlap between the two samples is currently small, it appears likely that the same subset of the field population is involved.

\section{Uncertainties in the Local Field Galaxy Luminosity Function}

Only recently has the form and absolute normalisation of the local field galaxy luminosity function (LF) been reliably determined. Efstathiou et al. (1988) analysed the DARS survey of 326 galaxies in 5 Schmidt-sized fields to $b_{J} \simeq 17$ demonstrating that a Schechter (1976) form is appropriate. Without correcting for photometric errors, they determined Schechter parameters $<M_{B}^{*}, \alpha, \Phi^{*}>$ of $<-19.7,-1.07,0.0156>\left(H_{o}=100 \mathrm{kms} \mathrm{sec}^{-1} \mathrm{Mpc}^{-1}\right)$. The more extensive panoramic sparse-sampled APM-Stromlo southern survey of 1769 galaxies (Loveday et al., 1992) found similar parameters <-19.7, -1.11, 
$0.0140>$. Both surveys to $b_{J}=17$ constrain the faint end slope of the local LF only to absolute magnitudes $M_{b J} \simeq$-16. An upturn at fainter luminosities such as that claimed for the Virgo cluster (Binggeli et al., 1988) cannot be formally ruled out. A local population of such feeble sources would have an Euclidean count slope and might dominate the faint counts diminishing any evolution that would otherwise be inferred (Kron, 1982). Furthermore, surveys limited at relatively bright apparent magnitudes adopt high surface brightness detection thresholds and may be poorly-suited for finding intrinsically faint galaxies (McGaugh, 1994). Clearly, the most reliable constraint on the faint end of the local LF comes from spectroscopy at those faint limits where the contribution can be directly measured (Glazebrook et al., 1994).

A related uncertainty which has plagued the subject concerns the question of the absolute normalisation of the LF. Although the DARS and APMStromlo surveys have consistent values of $\Phi^{*}$, the number counts steepen beyond their apparent magnitude limits, $17<b_{J}<20$, more than can be accounted for by non-evolving models, suggesting southern volumes with $b_{J}<17$ may be unrepresentative, or dramatic evolution in recent times (Maddox et al., 1990). The explanation for this anomaly remains unclear but in $\S 5$ we present further evidence that local volumes of radius $\simeq 200$ Mpc may be underabundant by a factor of $\simeq 2$.

\section{The Autofib Redshift Survey}

The disparate nature of "benchmark" $b_{J}<17$ redshift surveys and deep surveys within narrow faint apparent magnitude slices is not ideal. Broadhurst et al., Colless et al. and Glazebrook et al. were only able to compare the faint redshift distribution $N(z)$ with empirical predictions based on the bright survey. At no redshift was there a sufficient range in luminosity to examine the form of the LF directly. Moreover, the number of pencil beams sampled was relatively small ( $\simeq 5$ each) and some fields are heavily clustered raising the worry that sampling errors may affect the conclusions.

We have therefore conducted a new "Autofib" survey (using the robotic fibre positioner built for the Anglo-Australian Telescope - Parry and Sharples, 1988) spanning a wide apparent magnitude range in many directions and enabling direct reconstruction of the LF at various redshifts. It includes $\simeq 700$ redshifts from earlier magnitude-limited surveys and about 1000 new redshifts from Autofib contained within the intermediate magnitude range $17<b_{J}<22$ (see Table 1 ). Further details of the construction and analysis of this new survey are contained in Heyl's thesis (1994) and preliminary results are presented here, in Colless (1994) and Ellis et al. (1994). 
TABLE 1. The Autofib Redshift Survey (Ellis et al., 1994)

\begin{tabular}{lllll}
\hline Survey & $b_{j}$ limits & Area deg $^{2}$ & Fields & Redshifts \\
\hline DARS (Peterson et al., 1986) & $11.5-16.8$ & 70.80 & 5 & 326 \\
BES (Broadhurst et al., 1988) & $20.0-21.5$ & 0.50 & 5 & 188 \\
LDSS-1 (Colless et al., 1990,1993) & $21.0-22.5$ & 0.12 & 6 & 100 \\
Autofib bright & $17.0-20.0$ & 5.50 & 16 & 480 \\
Autofib faint & $19.5-22.0$ & 4.70 & 32 & 546 \\
LDSS-2 (Glazebrook et al., 1994) & $22.5-24.0$ & 0.07 & 5 & 73 \\
\hline TOTAL & & & & 1713 \\
\hline
\end{tabular}

A major difficulty in estimating absolute magnitudes of faint galaxies given a catalogue of individual $b_{J}$ magnitudes and redshifts is estimation of the $k$-correction which depends on the galaxy's (unknown) spectral energy distribution (SED). At a given redshift within the range sampled, $k_{b J}(z)$ changes by $\simeq 1$ magnitude across the Hubble sequence (c.f. King and Ellis, 1985) demonstrating the importance of inferring the SED of a given faint galaxy.

Broad-band colours are only available for a subset of the survey and represent a poor substitute for a proper spectral classification which will be useful in subsequent analyses. Heyl (1994) has devised a classifier based on cross-correlation of the faint spectra against the wide aperture local spectral catalogue of Kennicutt (1992). Knowing the Kennicutt morphology which best matches the faint galaxy, the $k$-correction is then determined with reference to King and Ellis' compilation. Realistic simulations based on Kennicutt's spectra which include photon noise and sky subtraction difficulties suggest the correct spectral class is returned to within \pm 1 class for $90 \%$ of the cases. 6 classes span the entire Hubble sequence.

One might worry that a class of galaxy exists at faint limits which is not represented in Kennicutt's list. To check this we devised an internal classification scheme based on the [O II] 3727 Å equivalent width and 4000 $\AA$ break. Coadding spectra categorised in a 2-dimensional scheme based on these indices, it is straightforward to identify the high $\mathrm{s} / \mathrm{n}$ composites with Kennicutt equivalents, suggesting no serious omissions. Indeed, with some restrictions, it is even possible to check the $k$-corrections directly by moving a $b_{J}$ filter over the coadded data (Heyl, 1994).

Most of the Autofib survey is redshift-complete at the $70-85 \%$ level. Across the 6 sub-surveys in Table 1, tests show that incompleteness is primarily a function of apparent magnitude arising from poor continuum $\mathrm{s} / \mathrm{n}$ rather than systematics which correlate with galaxy type. This can 


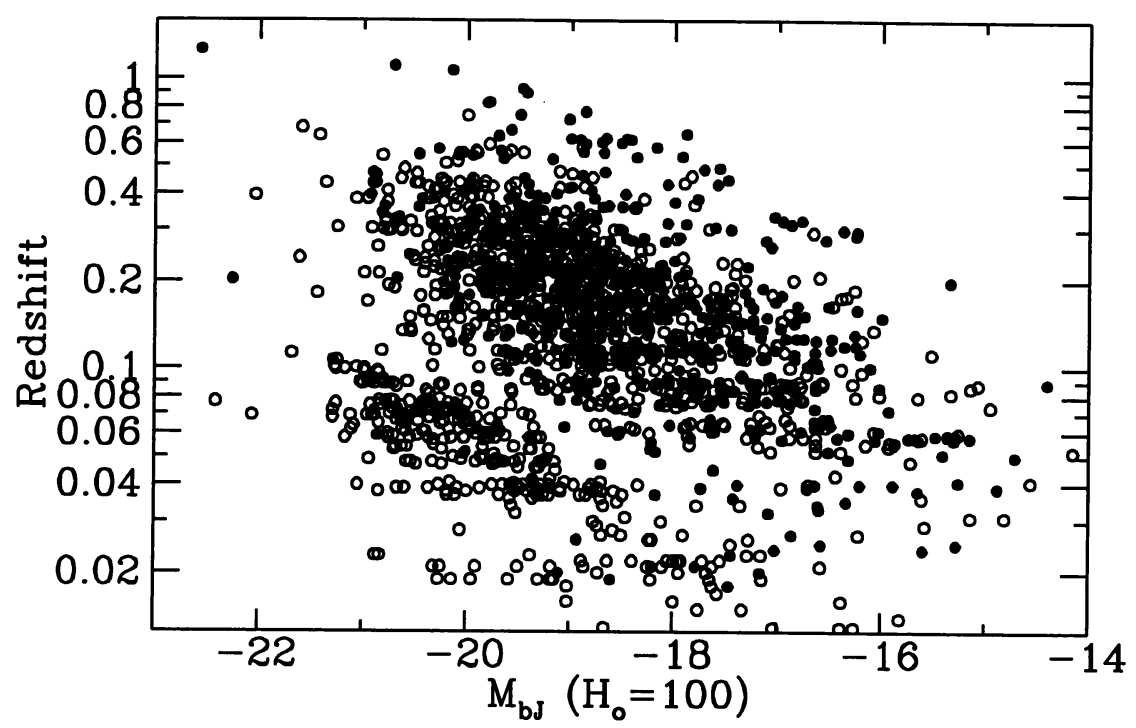

Figure 1. Absolute magnitudes and redshifts for the Autofib survey (Ellis et al., 1994). Filled symbols refer to galaxies with strong [O II] emission.

be checked by comparing $N(z)$ at the brighter (complete) end of the faint samples with that for the fainter (incomplete) end of the bright ones. By weighting in each sample according to the incompleteness at that apparent magnitude, we can recover satisfactory $V / V_{\max }$ distributions for each spectral class (Colless, 1994).

The distribution of absolute magnitudes and redshifts is shown in Fig. 1. Luminosity functions have been derived from this data using both traditional $1 / V_{\max }$ estimators with errors based on a bootstrap technique and a step-wise maximum likelihood method modified as described by Heyl (1994).

\section{Evolution of the Field Galaxy Luminosity Function}

The enlarged number of faint pencil beams in the Autofib survey leads to new constraints on the local LF as well as on its form at high $z .560$ galaxies in the survey have $0<z<0.1$ but few are less luminous than $\mathrm{M}_{b J} \simeq-16.0$; most with $b_{J}>22$ galaxies lie beyond $z \simeq 0.1$. The paucity of low luminosity galaxies severely limits the size of any possible upturn in the local LF to $M_{b J} \simeq-14$ (Fig. 2). As the volumes probed are now quite substantial and the photometric data used to select these galaxies penetrate to surface brightness limits below $\mu_{b J}=26.5 \operatorname{arcsec}^{-2}$, it becomes hard to argue that the flat LF is due to selection biases. The redshift distribution at $b_{J}=24$ 


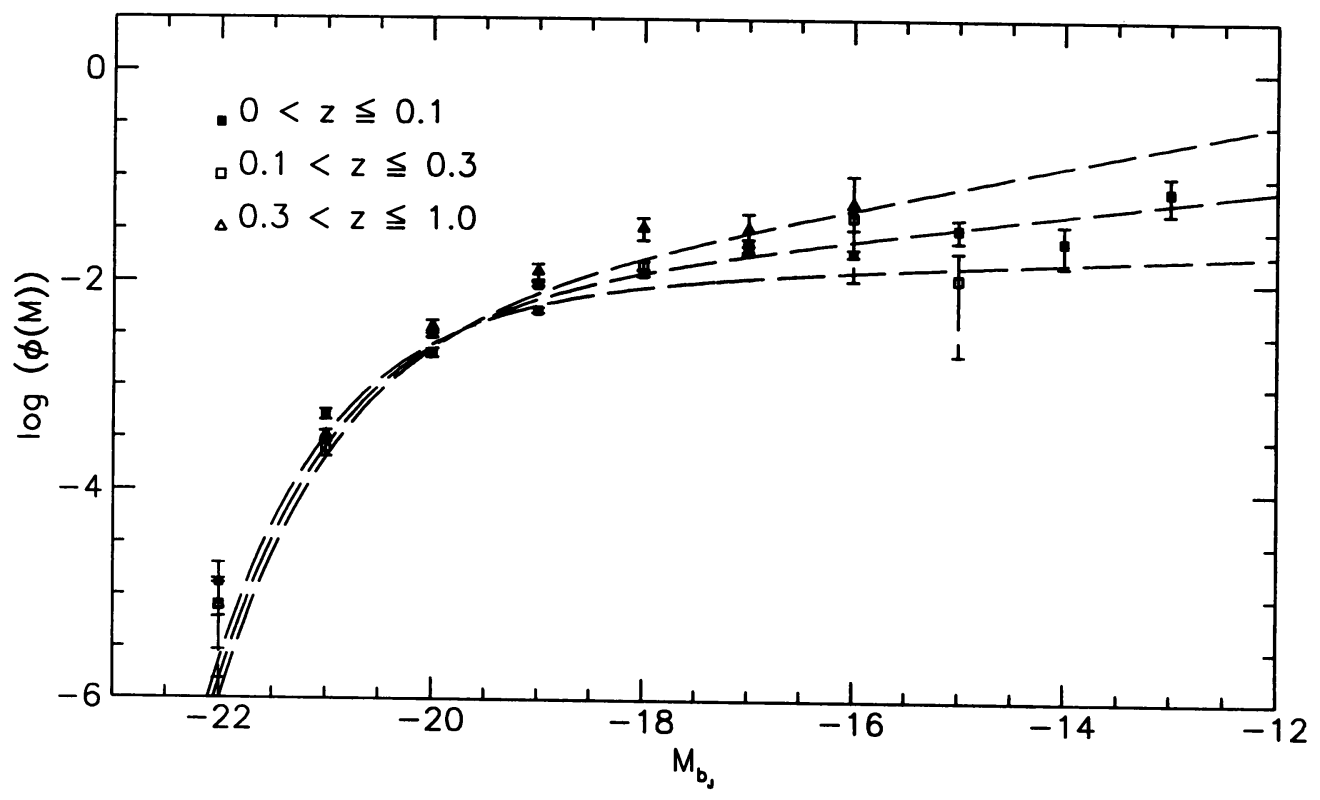

Figure 2. Luminosity functions from the Autofib survey (Ellis et al., 1994) in different redshift intervals. Lines refer to Schechter faint end slopes $\alpha=-1.1,-1.3$ and -1.5.

eliminates the possibility that the faint source counts are significantly contaminated by a population of low luminosity galaxies under-represented in the original $b_{J}<17$ surveys (Glazebrook et al., 1994).

Fig. 2 also shows a highly suggestive steepening of the faint end slope of the LF with increasing redshift. Formally, a change in shape with $z$ is significant at the $99.9 \%$ level. Less securely, there is no obvious brightening in the luminous end of the LF to $z \simeq 1$. The latter supports conclusions derived independently from massive galaxies identified on the basis of $\mathrm{Mg}$ II absorption lines seen in background QSO spectra (Steidel, 1994). A picture emerges whereby the LF is composed of two components - a luminous one evolving very slowly, if at all, over $z<1$ plus a rapidly-evolving component which decays dramatically in the sub-L* regime.

What physical parameters distinguish the galaxies that lie in these two components? The missing clue appears to be related to the star-formation rate (c.f. Fig. 1). Fig. 3 shows how sources with strong [O II] emission. contribute to the overall absolute magnitude distribution for the various redshift bins. The luminosity density of these star-forming galaxies has decayed by a factor $\simeq 10$ since $z \simeq 0.5$. 


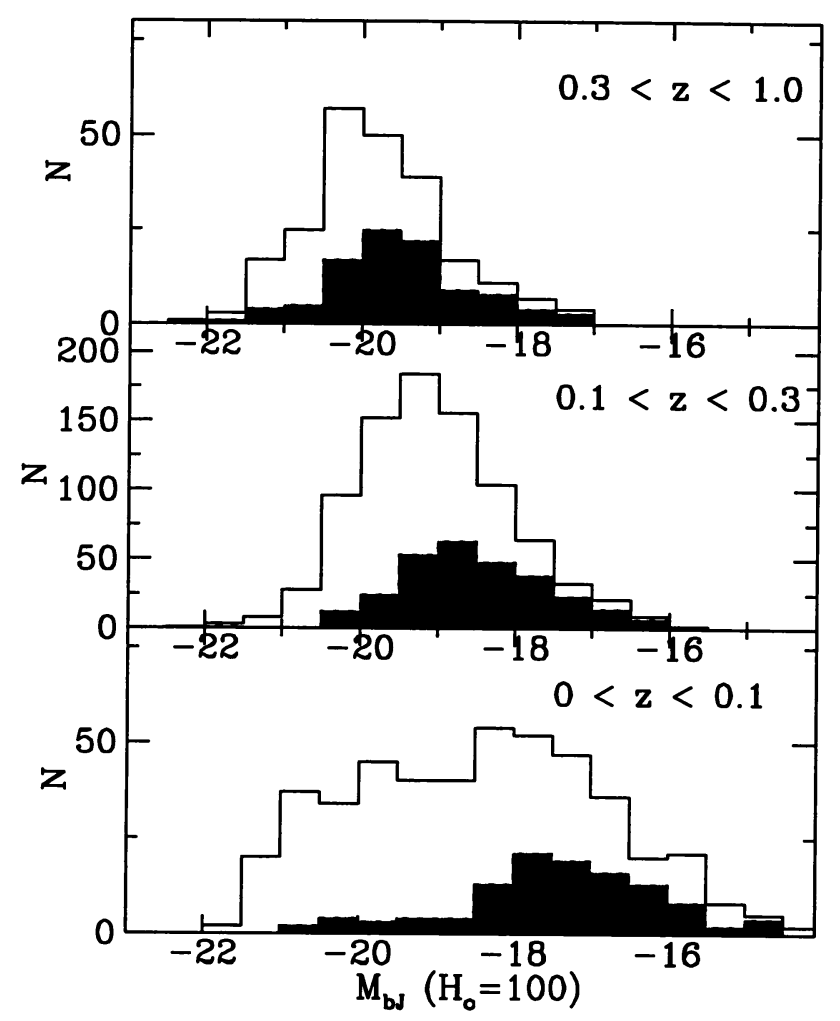

Figure 3. Absolute magnitude distributions for various redshift intervals. Shading refers to galaxies whose rest-frame [O II] $3727 \AA$ equivalent width exceeds $20 \AA$.

\section{Faint Galaxy Morphologies from HST}

The repair of HST promises to add a new dimension in the study of faint field galaxies. Early Cycle 4 images (Griffiths et al., 1994) have shown the ease with which resolved morphological features can be identified in $I \simeq 22$ galaxies. A new set of questions can be addressed with such data: (1) What is the morphological mixture of the high $z$ field population? (2) Are the faint blue galaxies a distinct morphological population? (3) Can we distinguish separate evolutionary trends for bulges and disks?

Ideally a large sample of HST morphologies with redshifts is required to address these issues. The largest collection of HST images is currently provided by the "Medium Deep Survey" (PI: Griffiths). In this key project, WFPC-2 is used in parallel mode associated with primary pointings defined by a variety of other observers. Redshifts have to be secured later. A limited amount of HST imaging has been done in the reverse mode: i.e. primary WFPC-2 imaging of fields with existing spectroscopy from the Aut$o f i b$ survey (PI: Broadhurst). Fig. 4 shows how powerful the combination of 


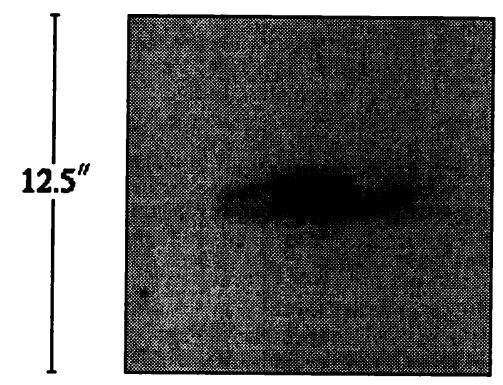

$B=23.7 \quad z=0.492$

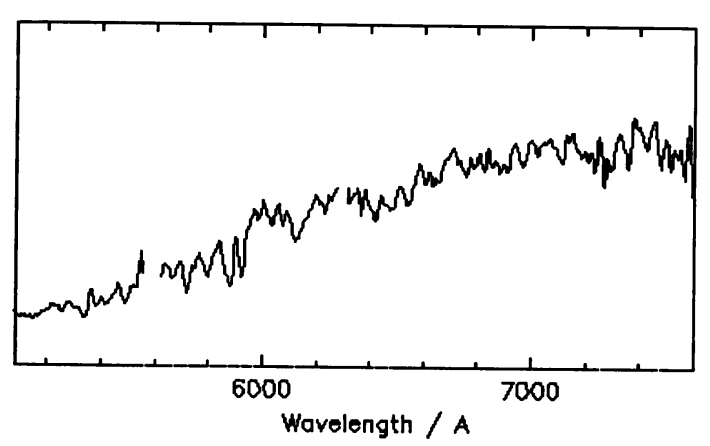

Figure 4. Ground-based spectrum and WFPC-2 image of a faint galaxy from the HST program of Broadhurst et al.. The late-type spectral class is confirmed morphologically.

ground-based spectroscopy and HST imaging will be. In a single WFPC-2 image $10 b_{J}<24$ galaxies from the LDSS-1/2 surveys reveal morphological types in excellent agreement with their spectral classes.

The most significant result to date comes from the Medium Deep Survey. Over $300 I<22$ galaxies have been classified on a simple E/S0: Spiral: Irr/Pec scheme (Glazebrook et al., 1995). The number-magnitude counts as a function of type are shown in Fig. 5 and illustrate that the spiral and early-type classes show little evidence for evolution. Significantly, both classes fit the predictions only if the absolute normalisation is $x \simeq 2$ higher than that derived from the 17th magnitude surveys (c.f. §2). On the other hand, the irregular and peculiar galaxies demonstrate a count slope much steeper than expected, consistent with significant evolution. Although the overlap with the spectral samples remains small, it seems highly likely that the [O II]-strong sources which decline dramatically in number since $z \simeq 1$ are the morphologically unusual examples in the HST samples. A morphologically-distinct population of sources appears to be responsible for the well-established excess population of faint blue galaxies.

Acknowledgements: The Autofib survey involves Matthew Colless, Tom Broadhurst, Jeremy Heyl and Karl Glazebrook and the Medium Deep Survey is led by Richard Griffiths. I thank all co-workers for allowing me to present data prior publication. I acknowledge financial support from the IAU and the assistance of Jacqueline Bergeron and Piet van der Kruit. 

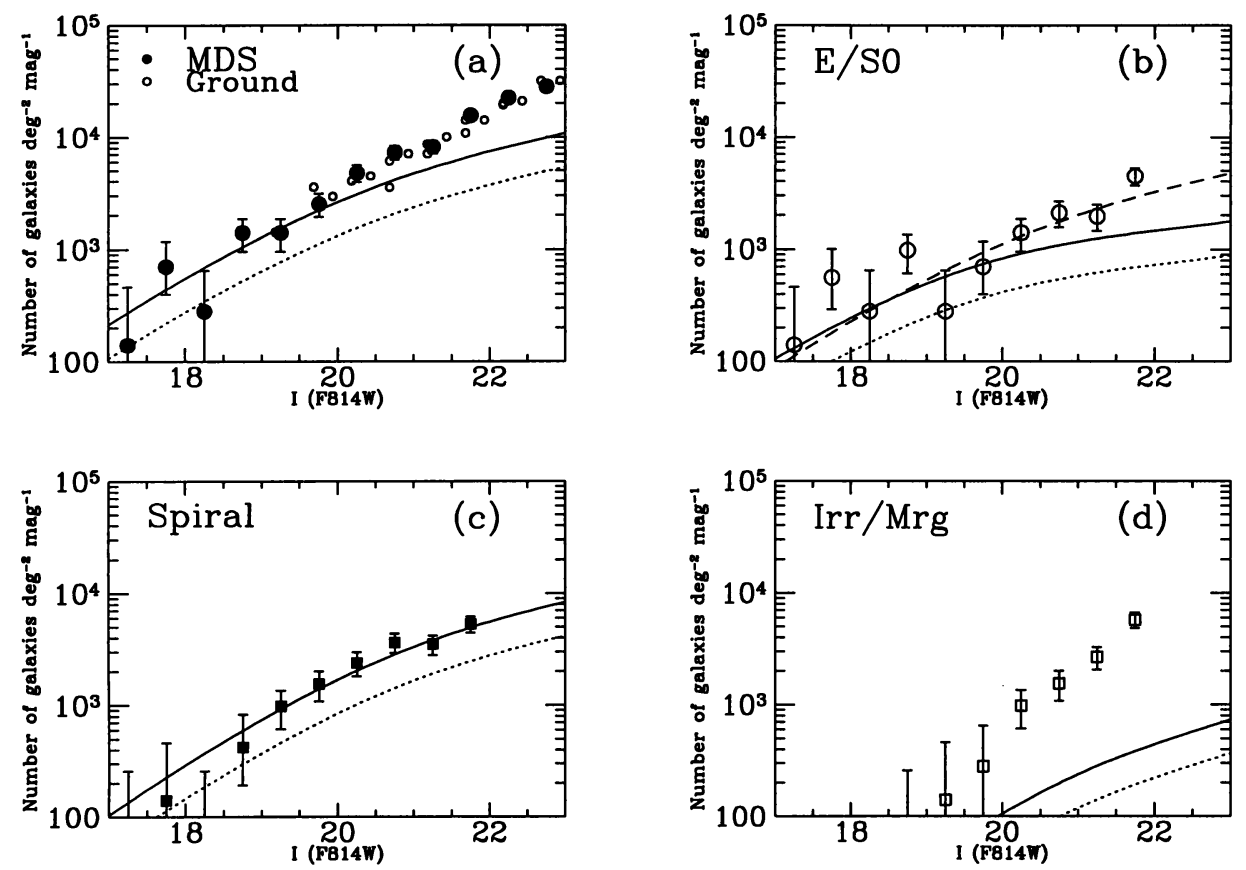

Figure 5. Morphological counts from the HST Medium Deep Survey (Glazebrook et al., 1995). Those of "regular" Hubble types are consistent with no evolution but irregular/peculiars show a steep slope suggesting rapid evolution.

\section{References}

Babul, A. and Rees, M.J., 1992, M. N. R. A. S. 255, 346

Binggeli, B., Sandage, A. and Tammann, G., 1988, Ann. Rev. Astron. Astroph. 26, 509

Broadhurst, T.J., Ellis, R.S. and Shanks, T., 1988, M. N. R. A. S. 235, 827

Broadhurst, T.J., Ellis, R.S. and Glazebrook, K., 1992, Nature 355, 55

Colless, M., Ellis, R.S., Taylor, K. and Hook, R., 1990, M. N. R. A. S. 244, 408

Colless, M., Ellis, R.S., Broadhurst, T.J., Taylor, K. and Peterson, B.A., 1993 M. N. R. A. S. 261, 19

Colless, M., Schade, D., Broadhurst, T.J. and Ellis, R.S., 1994, M. N. R. A. S. 267, 1108

Colless, M. Wide Field Spectroscopy, eds. Maddox, S.J. and Aragón-Salamanca, A., World Scientific, in press

Cowie, L., Songaila, A. and Hu, E.M., 1991, Nature 354, 460

Efstathiou, G., Ellis, R.S. and Peterson, B.A., 1988, M. N. R. A. S. 232, 431

Ellis, R.S., Broadhurst, T.J, Colless, M.M, Heyl, J.S. and Glazebrook, K., 1994, M. N. R. A. S., submitted

Giraud, E., 1992, A. \& A. 257, 501

Glazebrook, K., Ellis, R.S., Colless, M., Broadhurst, T.J., Allington-Smith, J. and Tanvir, N., 1994, M. N.R.A.S., in press

Glazebrook, K., Ellis, R.S., Santiago, B. and Griffiths, R., 1995, Nature, submitted

Griffiths, R.E. et al., 1994) Ap. J. 435, L19

Heyl, J., 1994 M.Sc. thesis, University of Cambridge, UK

Kennicutt, R. C., 1992, Ap. J. 388, 310

King, C.R. and Ellis, R.S., 1985, Ap. J. 288, 456 
Kron, R., 1982, Vistas Astron. 26, 37

Loveday, J., Peterson, B.A., Efstathiou, G., Maddox, S.J. and Sutherland, W.J., 1992, Ap. J. 390, 338

Maddox, S.J., Sutherland, W.J., Efstathiou, G., Loveday, J. and Peterson, B.A., 1990, M. N. R. A. S. 247, $1 \mathrm{P}$

McGaugh, S., 1994, Nature 367, 538

Parry, I.R. and Sharples, R.M., 1988, Fiber Optics in Astronomy, ed. Barden, S.M., PASP series Vol. 3, p93

Steidel, C., 1994, Wide Field Spectroscopy, eds. Maddox, S.J. and Aragón-Salamanca, A., World Scientific, in press

RICH: Do you get any sense of whether the field or clusters have a greater fraction of peculiar or unclassifiable objects?

ELLIS: A connection between the puzzling increase in blue starforming field galaxies with redshift and the Butcher-Oemler effect in $\mathrm{z} \approx 0.4$ clysters is an interesting possibility. The firste WFPC- 2 images of $z \approx 0.4$ clusters do show many of the same usual types of galaxies, as I showed in the field. However, it must be remembered that cluster membership has not been determined exhaustively in these clusters to the HST magnitude limits. It is too early to draw any physical conclusions on any similar behavious except to note that interesting affects are inevitable if gas-rich field galaxies fall into rich clusters.

FABER: In one slide you showed that the number of galaxies per unit volume at the bright end of the luminosity function was the same at $\mathrm{z}>0.5$, but later you said that the volume density -even of normal objects- was a factor of two higher at the same z's. How do you reconcile these two?

ELLIS: The high-z luminosity function (LF) from our redshift survey does tentatively allow some number evolution or brightening at $\mathrm{z}>0.5$. The vertical adjustment I made in predicting the Medium Deep Survey morphological distribution was arbitrary and reflects soem acknowledgement that the absolute normalisation $\Phi^{*}$ of the LF could still be uncertain. At this stage, with only 10 MDS fields, not too much emphasis should be placed on the absolute shift. 